\title{
Desarrollo y evaluación de la expresión oral a través de las Tecnologías de la Relación, Información y Comunicación (TRIC) en la enseñanza y aprendizaje de español como lengua extranjera.
}

\author{
Development and Assessment of Oral Expression Through Relation, Information and \\ Communication Technologies (RICT) in the Teaching and Learning of Spanish as a Foreign \\ Language
}

Juan C. Rocha-Osornio ${ }^{a}$

\begin{abstract}
:
The development and assessment of oral expression in the teaching of Spanish as a Foreign Language (SFL) represents one of the greatest challenges that teachers and students face. Numerous research studies reveal the need to pay closer attention to the affective dimension of learning to reduce feelings of anxiety that often lead learners to internalize negative and damaging emotions when it comes to acquiring a foreign language. Fortunately, with the advent of Information and Communication Technologies (ICT), it has become possible to make way for new digital tools that seek to provide students with a positive and meaningful experience, while at the same time teachers go beyond traditional methodologies that have dominated the field of foreign language teaching (FLT) for decades. However, its use has been limited in terms of the role and treatment of the affective dimension, which is why this article proposes the incorporation of Relation, Information and Communication Technologies (RICT) as a way to encourage autonomous and experiential learning increasingly demanded by the particular needs and context of a new generation of digital native learners in the XXI century. To contextualize the use of RICT, we present a didactic activity that consists in making short video clips in SFL courses in the Department of Spanish \& Portuguese at the University of Toronto, Canada.
\end{abstract}

\section{Keywords:}

Affective Dimension, Digital Competency, Linguistic Anxiety, Oral Expression; Relation, Information and Communication Technologies (RICT, Spanish as a Foreign Language

\section{Resumen:}

El desarrollo y la evaluación de la expresión oral en la enseñanza y aprendizaje de Español como Lengua Extranjera (ELE) representa uno de los mayores retos que los docentes y alumnos enfrentan. Numerosos estudios revelan la necesidad de poner más atención a la dimensión afectiva como una forma de reducir la ansiedad que suele conducir a los aprendientes a internalizar emociones negativas y perjudiciales en la adquisición de una lengua extranjera. Afortunadamente, con la llegada de las tecnologías de la información y la comunicación (TIC), se ha podido dar paso a nuevas herramientas digitales que buscan dotar al alumnado de una experiencia positiva y significativa, al mismo tiempo que los docentes van más allá de los confines tradicionales que por décadas han dominado el campo de la enseñanza de lenguas extranjeras (LE). Sin embargo, su uso ha resultado limitado en cuanto al rol y tratamiento de la dimensión afectiva, por lo que en este artículo se propone la incorporación de las Tecnologías de la Relación, Información y Comunicación (TRIC), como una manera de fomentar el aprendizaje autónomo y experiencial cada vez más demandado por las necesidades y contexto particulares de la nueva generación de aprendientes nativos tecnológicos del siglo XXI. Para contextualizar el uso y aplicación de las TRIC, presentamos una actividad didáctica que consiste en la realización de videos cortos en los cursos de ELE en el departamento de español y portugués de la Universidad de Toronto, Canadá.

\section{Palabras Clave:}

Ansiedad lingüística, competencia digital, dimensión afectiva, español como lengua extranjera, expresión oral; tecnologías de la relación, información y comunicación (TRIC),

\section{INTRODUCCIÓN : EL TRÁNSITO DE LO ANÁLOGO A LO DIGITAL}

Uno de los mayores retos en la enseñanza y aprendizaje de lenguas extranjeras (en adelante LE) es desarrollar y evaluar de manera efectiva (y afectiva) la expresión oral de nuestros

\footnotetext{
${ }^{a}$ Corresponding author, University of Toronto, https://orcid.org/0000-0002-4759-5000, Email: juancarlos.rochaosornio@utoronto.ca
} 
estudiantes, sobre todo en la actualidad con el pujante uso de la tecnología y su vasto abanico de posibilidades. Fomentar la expresión oral que conlleve a un buen nivel de competencia comunicativa, ha sido ampliamente documentado por docentes e investigadores como un proceso difícil de llevar a cabo, debido a la ansiedad que muchas veces suelen experimentar los alumnos, y que les puede ocasionar sentirse nerviosos, vulnerables y poco seguros de sí mismos, particularmente al hablar en público o llegado el momento de ser evaluados.

Desafortunadamente, las emociones negativas pueden provocar que el proceso de aprendizaje de una LE se vea empañado y que, por paradójico que pueda parecer, se llegue también a desarrollar un hábito nocivo asociado con la lengua que se busca aprender: "the student begins to expect to be nervous and perform poorly" (MacIntyre \& Gardner, 1991, p. 10). De manera similar, otros autores concluyen que la destreza oral por medio de la práctica y evaluación en clase (O’Sullivan et al., 2006), así como su medición y efectividad a través de la tecnología (Levy \& Stockwell, 2006), suele traer consigo desafíos importantes que los docentes debemos considerar cuidadosamente. Al respecto, Kremers (2000) ofrece una pertinente reflexión: "la expresión oral es la destreza que al mismo tiempo más nos interesa y, sin embargo, más nos cuesta dominar en una lengua extranjera. En el aula se ve reflejada esta paradoja por la actitud reservada tanto de los profesores como de los alumnos a la hora de practicarla" (p. 461).

Como vemos, estamos ante un proceso complejo que se hace patente en ambas partes del binomio enseñanza-aprendizaje, por lo que abordarlo desde una perspectiva transdisciplinaria enfocada en un carácter didáctico e interactivo de la lengua, colaborativo y cooperativo facilitará mejor dicho proceso. Adoptar este camino nos permitirá a los docentes ajustarnos mejor a las necesidades específicas de nuestros estudiantes, mediante el diseño de actividades significativas y un sistema de evaluación que verdaderamente potencie su destreza oral con soltura. Al mismo tiempo se intenta dejar de lado la presión a equivocarse o a pensar que se está haciendo el ridículo.

El año 2007 marca un hito importante en la revolución tecnológica -o "tecnocientífica" en palabras de Echeverría (2003) - con la salida al mercado de los primeros teléfonos móviles inteligentes con pantalla de tacto, como el iPhone de la reconocida marca Apple. A partir de entonces, miles de recursos didácticos, ejercicios, actividades y tareas que ya estaban disponibles a través de herramientas Web 2.0 (conocida también como la Web social o la Web colaborativa), se adaptan a un nuevo ambiente más cómodo y versátil (el M-learning $o$ Mobile Learning), para convertirse en un aliado importante de la comunidad docente dispuesta a ir más allá de los confines tradicionales en la enseñanza de una LE. El M-learning propone un aprendizaje por medio de aplicaciones móviles. Precisamente, en ese mismo año, el uso de las Tecnologías de la Información y la Comunicación (en adelante TIC), aplicado al contexto educativo se convierte en un referente importante y pasa a formar parte medular de programas europeos como Comenius, Erasmus, Leonardo da Vinci y Grundtvig. En este sentido, se evidencia también un interés por incorporar las TIC en la formación docente y de esta manera acrecentar el saber y conocimiento digitalizado; herramientas esenciales de las competencias del siglo XXI que harán más efectivo el proceso de enseñanza-aprendizaje (Iglesias, 2016, p. 75). El propio Plan Curricular del Instituto Cervantes (2006) enfatiza la importancia que los docentes deben dar a incorporar las diferentes facetas de la competencia digital en su profesión: "Implica el uso confiado y crítico de los medios electrónicos para el trabajo, ocio y comunicación". A la postre, el Marco Común de Competencia Digital Docente (2017, p. 69), ofrece una detalladísima descripción y contexto de aplicación de las tecnologías digitales a través de varios fines que bien pueden servir como catalizador del elemento creativo en una clase de LE, tal y como se expone a continuación:

- Ocio: utilización de las tecnologías para cuestiones personales o de entretenimiento (los ejemplos incluyen: búsqueda de vuelos para vacaciones, juegos, lectura de libros digitales, visionado de vídeos en directo en la web, escucha de música a través de herramientas digitales).

- Social: interacción con amigos y compañeros con herramientas digitales (los ejemplos incluyen: envío de correos electrónicos o sms, participación en redes sociales, vinculación con otros a través de comunidades en red.

- Transacciones comerciales: utilización de recursos en línea para comprar y vender bienes, comercio electrónico, consumo en línea (los ejemplos incluyen: compra en línea de un billete de avión o de tren, compra de aplicaciones y software, compra y venta de bienes virtuales, como los elementos utilizados en mundos virtuales de entornos de videojuegos, participación en servicios de cliente a cliente.

- Aprendizaje: utilización de las tecnologías para el aprendizaje a lo largo de la vida (los ejemplos incluyen: el uso de software de referencia cuando se escribe un trabajo universitario, utilización de la Web para la búsqueda de información, uso de suscripciones especializadas para acceder a artículos científicos, utilización de comunidades en línea, como una red de intercambio).

- Empleo: utilización de tecnologías para realizar diferentes tipos de trabajo (los ejemplos incluyen: el uso de software para registrar los pedidos de los clientes en un bar y calcular la cuenta, utilización de hojas de cálculo para calcular un presupuesto, comprensión de la configuración inalámbrica de máquinas mecánicas). 
- Ciudadanía: utilización de las tecnologías para formar parte activa de la vida cívica y utilizar los servicios (los ejemplos incluyen: banca en línea, administración electrónica y comercio electrónico).

- Bienestar: uso de las tecnologías para fines relacionados con la salud (los ejemplos incluyen: consultas médicas, revisión de información en red para cuestiones relacionas con la salud, utilización de un rastreo de datos de registro del sistema acerca de actividades deportivas).

Actualmente, el aprendizaje en entornos virtuales se encuentra en una etapa de expansión que apela a las necesidades de un estudiantado que vislumbra la presencia mayoritaria en clase de los llamados "nativos tecnológicos", término utilizado por Marc Prensky (2001) en su célebre artículo On the Horizon, para denominar a la generación que nació y creció con el desarrollo de las TIC. Años después, Prensky aludiría a los “inmigrantes digitales" para demostrar que "están empleando una lengua obsoleta (la propia de la edad pre-digital) para instruir a una generación que controla perfectamente dicha lengua" (Prensky, 2010, p. 6). Por esta razón, proveer a los nativos tecnológicos de las herramientas digitales que precisan para gestionar su propio aprendizaje, será uno de los quehaceres fundamentales de los docentes en esta nueva era tecnológica.

Afortunadamente, los beneficios de emplear la tecnología son varios; como el aspecto ligado a la motivación extrínseca, ya que "el uso adecuado de las TIC incrementa la motivación de los estudiantes al proporcionarles un control más personal e individualizado sobre su propia experiencia de aprendizaje, lo que puede afectar positivamente al rendimiento académico" (Iglesias, 2016, p. 75). Por su parte, Tesouro y Puiggalí (2004) señalan que la incursión de recursos digitales puede ayudar a los estudiantes a reducir la ansiedad; Sloep y Berlanga (2011) resaltan el aspecto de la cooperación; Fandos y Silvestre (2011) aluden a que fomenta mejor la creatividad y el pensamiento crítico; Ezeiza y Palacios (2009) destacan la conciencia multicultural; y Aguilar y Leiva (2012), por su parte, ahondan en que la digitalización puede facilitar el proceso de autoevaluación. Pero también hay dificultades, como el poco o nulo acceso a los recursos digitales fuera del aula, según Espinar y López (2009); un sentir de soledad y la inhabilidad de no poder distinguir entre fuentes falsas y confiables (Vilchez 2005); la adicción a los medios digitales, según Pérez (2010); y la distracción y pérdida de la concentración como señalan Caro y Plaza (2016).

En el caso particular de la enseñanza y aprendizaje de Español como Lengua Extranjera (ELE en adelante), la posibilidad de desarrollar y gestionar cursos de alto impacto basados en un acercamiento vigostkiano (interaccionismo), se hace aún más necesaria en la actual era digital. En los últimos años el concepto de las TIC ha venido dejando de lado un aspecto fundamental sin el cual el papel de los estudiantes y la dinámica generada en clase no son aprovechados al máximo, pues no solamente se trata de implementar muestras de tecnología superficiales, como el uso del libro de texto adaptado al formato electrónico (e-book), o el uso de presentaciones PowerPoint, por mencionar solo algunos de los ejemplos más comunes. Por tanto, en este artículo se propone la aplicación de las Tecnologías de la Relación, Información y Comunicación (TRIC), término acuñado por Aranda et al., (2012) con el objetivo de renovar e ir más allá del concepto TIC. De manera particular, se considera la dimensión afectiva; es decir, la importancia que tienen las emociones en el proceso de enseñanza-aprendizaje de una LE. Para este fin, se propone una actividad didáctica cimentada en el uso y aplicación de las TRIC a través de la realización de videos cortos en clase, y llevada a cabo en los cursos de lengua española del departamento de español y portugués de la Universidad de Toronto, Canadá, desde el año 2017 al presente. De manera particular, se intenta ayudar a reducir el filtro afectivo que, en muchos casos, es capaz de elevar los niveles de ansiedad de los aprendientes y con ello afectar su desempeño y autoconfianza de expresarse oralmente en la lengua meta. Por esta razón, es importante buscar alternativas a los medios tradicionales para que con ello se haga del estudiantado partícipe activo del aprendizaje autónomo y experiencial, tal y como lo propone el Plan Curricular del Instituto Cervantes. Así, se fomentará un ambiente positivo en clase que permitirá desarrollar y evaluar el componente de expresión oral de una manera más efectiva y sin olvidar el aspecto afectivo. Por último, se deja constancia de que este trabajo es de carácter descriptivo y de análisis reflexivo con la intención de que otros docentes de LE lo consideren y, si así lo quisiesen, adapten a sus propias necesidades y/o contextos locales de enseñanza.

\section{USO Y APLICACIÓN DE LAS TIC EN EL NUEVO AMBIENTE DIGITAL EDUCATIVO}

Como resultado de la nueva era digital que inevitablemente se postra ante nosotros, se cuestiona cada vez más el proceso de aprendizaje apoyado en las teorías conductistas de las cuales han derivado la mayoría de las estrategias tradicionalistas de enseñanza, y que por décadas se han utilizado en una clase presencial de LE. Bajo esta tónica, ha sido común el uso de los medios didácticos como la pizarra y el libro (o manual de trabajo) cuyo uso, en muchos casos y dependiendo de la institución donde se labore, puede llegar a ser obligatorio. También, se ha observado una tendencia a la estructuración de clases a partir de contenidos lingüísticos y funcionales que deben seguirse uniformemente. En términos del alumno, se ha confiado en que éste asimile de manera mecánica y refuerce sus conocimientos a través de ejercicios y tareas primordialmente hechos a base de papel. Básicamente y como subraya Veuthey (2008) sobre esta concepción conductista del aprendizaje, "el alumno tiene un papel relativamente pasivo, su misión consiste en acumular los conocimientos expuestos por el profesor, que 
es quien dirige el aprendizaje" (p. 62). En términos concretos, dicha atmósfera dista bastante de ser ideal, ya que poco hace para generar un ambiente de aprendizaje autónomo y compatible con la manera de ser del aprendiente y nativo tecnológico; quien ocupa cada vez más un lugar mayoritario en nuestras clases, sin importar la modalidad: presencial o virtual.

Para enmarcar el tránsito de la educación tradicional a la actual o emergente, Caballero (2009) propone la siguiente tabla:

\begin{tabular}{|c|c|c|}
\hline ESCUELA & TRADICIONAL & $\begin{array}{l}\text { ACTUAL- } \\
\text { EMERGENTE }\end{array}$ \\
\hline $\begin{array}{l}\text { Formatos de } \\
\text { conocimiento. }\end{array}$ & $\begin{array}{l}\text { El libro, } \\
\text { enciclopedias. }\end{array}$ & $\begin{array}{l}\text { DVD, bibliotecas } \\
\text { virtuales, videos, } \\
\text { portafolios } \\
\text { digitales, blogs, } \\
\text { wikis, web 2.0. }\end{array}$ \\
\hline $\begin{array}{l}\text { Transmisión } \\
\text { de } \\
\text { conocimientos. }\end{array}$ & $\begin{array}{l}\text { El conocimiento es } \\
\text { transmitido en una } \\
\text { relación jerárquica } \\
\text { del saber, la lectura } \\
\text { lineal. El contenido } \\
\text { es fijo. El alumno es } \\
\text { ajeno al } \\
\text { conocimiento, su } \\
\text { recorrido ya está } \\
\text { prefijado. }\end{array}$ & $\begin{array}{l}\text { El conocimiento } \\
\text { es transmitido en } \\
\text { una relación } \\
\text { asociativa del } \\
\text { saber, mediante el } \\
\text { hipertexto, al cual } \\
\text { se accede a través } \\
\text { de las redes y } \\
\text { nuevos formatos } \\
\text { multimedia } \\
\text { (sonido, texto, } \\
\text { imagen, video). } \\
\text { El contenido y su } \\
\text { recorrido son } \\
\text { cambiantes, } \\
\text { dinámicos e } \\
\text { interactivos. El } \\
\text { sujeto de } \\
\text { aprendizaje } \\
\text { escoge la ruta a } \\
\text { utilizar y decide } \\
\text { sobre aquellos } \\
\text { contenidos de su } \\
\text { interés. }\end{array}$ \\
\hline $\begin{array}{l}\text { Enfoque o } \\
\text { método de } \\
\text { construcción, } \\
\text { apropiación, } \\
\text { manejo y } \\
\text { delimitación } \\
\text { de los } \\
\text { contenidos y } \\
\text { saberes. }\end{array}$ & $\begin{array}{l}\text { El conocimiento se } \\
\text { maneja por } \\
\text { disciplinas } \\
\text { reduccionistas, se } \\
\text { niega la } \\
\text { complejidad. } \\
\text { Abordaje de saberes } \\
\text { fragmentados, } \\
\text { muchas veces } \\
\text { descontextualizados, }\end{array}$ & $\begin{array}{l}\text { El acceso al } \\
\text { conocimiento es } \\
\text { transdisciplinario; } \\
\text { se asume que el } \\
\text { conocimiento es } \\
\text { complejo, tejido } \\
\text { en conjunto, por } \\
\text { tanto, no puede } \\
\text { separarse en } \\
\text { compartimentos }\end{array}$ \\
\hline
\end{tabular}

\begin{tabular}{|c|c|c|}
\hline & $\begin{array}{l}\text { tanto del sujeto de } \\
\text { aprendizaje, como el } \\
\text { objeto de estudio. El } \\
\text { conocimiento } \\
\text { enciclopédico se } \\
\text { asume como verdad, } \\
\text { de acuerdo a los } \\
\text { supuestos del } \\
\text { método científico. }\end{array}$ & $\begin{array}{l}\text { estancos, lo que } \\
\text { implica manejo de } \\
\text { diversidad de } \\
\text { saberes vinculados } \\
\text { a un tema } \\
\text { particular. }\end{array}$ \\
\hline $\begin{array}{l}\text { Relación } \\
\text { docente- } \\
\text { alumno. }\end{array}$ & $\begin{array}{l}\text { Relación autoritaria, } \\
\text { el docente es quien } \\
\text { sabe y enseña, el } \\
\text { alumno es quien } \\
\text { aprende. }\end{array}$ & $\begin{array}{l}\text { Relación de } \\
\text { facilitador entre } \\
\text { profesores y } \\
\text { alumnos; varios } \\
\text { factores } \\
\text { vinculados a un } \\
\text { tema. Todos son } \\
\text { sujetos de un } \\
\text { aprendizaje } \\
\text { común. }\end{array}$ \\
\hline $\begin{array}{l}\text { Tipo de } \\
\text { comunicación. }\end{array}$ & $\begin{array}{l}\text { Comunicación } \\
\text { únicamente } \\
\text { presencial. }\end{array}$ & $\begin{array}{l}\text { Comunicación } \\
\text { deslocalizada. Los } \\
\text { e-learning } \\
\text { apoyados en } \\
\text { videoconferencias, } \\
\text { aulas virtuales, } \\
\text { chats, forums. }\end{array}$ \\
\hline $\begin{array}{l}\text { Organización } \\
\text { de los diseños } \\
\text { curriculares } \\
\text { en torno a } \\
\text { problemas de } \\
\text { estudio. }\end{array}$ & $\begin{array}{l}\text { El contenido } \\
\text { curricular está } \\
\text { planificado de } \\
\text { acuerdo a materias. } \\
\text { Las soluciones están } \\
\text { dadas. En su } \\
\text { defecto, la } \\
\text { metodología parte } \\
\text { de un objeto } \\
\text { previamente } \\
\text { definido. }\end{array}$ & $\begin{array}{l}\text { El contenido } \\
\text { curricular se } \\
\text { ordena de acuerdo } \\
\text { a proyectos. } \\
\text { Facilita la } \\
\text { exploración, } \\
\text { permite la } \\
\text { simulación y el } \\
\text { aprender } \\
\text { haciendo. }\end{array}$ \\
\hline
\end{tabular}

Figura 1. Tránsito de la escuela.

Pero independientemente de cuáles sean los medios didácticos que utilicemos en clase, o las teorías de aprendizaje que nos parezcan adecuadas, es importante que los docentes de LE nos esmeremos en cumplir con las tareas y cualidades personales que propone Román-Mendoza (2018, p. 86), y que se muestran mediante el siguiente esquema donde se resalta la importancia de las habilidades interpersonales y la inteligencia emocional: 


\begin{tabular}{|l|}
\hline$\bullet \quad$ MOTIVA \\
\hline Enseña y aprende a aprender $=$ humanidad \\
\hline Escucha e interactúa $=$ sentido del humor \\
\hline Es exigente y flexible a la vez $=$ ejemplaridad \\
\hline$\bullet \quad$ NO DEJA DE FORMARSE \\
\hline Actualiza conocimientos $=$ competencia \\
\hline Aprende de sus compañeros $=$ credibilidad \\
\hline Actúa con principios $=$ ética \\
\hline$\bullet \quad$ SE ADAPTA \\
\hline A su alumnado $=$ interés y empatía \\
\hline A su entorno docente $=$ atención \\
\hline A sus propias actitudes y limitaciones $=$ honestidad \\
\hline \\
Trabaja PREPARA Y COLABORA \\
\hline Comparte materiales e ideas $=$ dedicación \\
\hline Actúa de mentor $=$ colegialidad \\
\hline
\end{tabular}

Figura 2. Tareas y cualidades personales del profesor de $L E / L 2$

En base a este esquema, vemos que el proceso de enseñanzaaprendizaje se aleja de considerar al docente como un mero acumulador y presentador de conocimientos. Es decir, aprender una LE no implica que el docente no pueda o deba aprender de sus alumnos; todo lo contrario, pues es precisamente en esa simbiosis donde resulta "fundamental que el profesor de L2 sepa crear una atmósfera traslúcida y de confianza en la que el alumno se sienta cómodo y se cultive, por parte de ambos, el "saber ser" (Roman-Mendoza, 2018, p. 85). El "saber ser" se refiere a la dimensión afectiva (a la importancia de las emociones), un aspecto que en los últimos años ha cobrado vigencia en el campo de LE: "un buen profesor de idiomas sabe y hace, pero esencialmente es" (en Arnold 2000, p. 22).

Para poder seguir enmarcando el tránsito de lo análogo a lo digital y sus repercusiones, conviene mencionar un reciente artículo sobre la enseñanza de ELE en línea como resultado de la emergencia sanitaria por COVID-19 en China. Este contexto resulta relevante en el ámbito de la enseñanza y aprendizaje de LE, pues el virus prácticamente se ha extendido por todos los rincones del mundo y ocasionado que muchos docentes hayamos tenido que mudar nuestras aulas al ambiente virtual. En el artículo, la profesora Liu Liu (2020) de la universidad de Estudios Extranjeros de Guangdong, Guangzhou, señala que "las modalidades tradicionales de enseñanza ya no pueden satisfacer la necesidad de las nuevas generaciones, ya que todos están en una era de información explosiva" (p. 2); no obstante, recomienda cautela con la suplantación del "modelo pedagógico tradicional" debido a que "los docentes deben prestar más atención a cómo interactuar con los alumnos y cómo estimular el aprendizaje activo de ellos al diseñar los cursos" (p. 3). Visto así, la autora nos invita claramente a no desatender el aspecto de la interacción con nuestros alumnos en el mundo educativo digital, pues existe el riesgo latente de poner más atención a la tecnología como un fin en sí mismo, que al proceso de aprender una LE mediante la interacción y el trabajo colaborativo con otros. De manera similar, estas palabras hacen eco de la explicación que Caballero (2009) provee para abordar tres supuestos:

1) Con la digitalización ya no se lee, ni se escribe igual

2) Con la digitalización ya no se enseña, ni se aprende igual

3) Un salto ético en la escuela sobre la base de la integración, de la escuela a la comunidad a partir de la emergencia de nuevas socialidades

Al analizar la explicación del segundo supuesto, se alude nuevamente a la imperiosa necesidad de modificar el rol del docente y que de orador pase a facilitador; pues solo de esta manera se conseguirá edificar el conocimiento de forma conjunta e integral, principal catalizador de un bien común: "En otras palabras, él se convierte en parte activa dentro del proceso de aprendizaje" (1). Desde esta vertiente, el antiguo papel del profesor como principal transmisor de conocimientos se vuelve obsoleto, sobre todo en la era digital, y en su lugar debe involucrarse activamente en el proceso de aprendizaje de sus alumnos. Propiciar y lograr una interacción positiva conducirá a que el desarrollo de la expresión oral y la evaluación de la competencia comunicativa no se adopte tan solo como un requisito más a satisfacer en un curso tradicional de LE, sino que los docentes pensemos cuidadosamente en las posibles repercusiones pedagógicas que nuestros criterios de evaluación habrán de tener en la calidad y aprovechamiento de nuestros cursos.

\section{LOS BENEFICIOS DE LAS TIC}

Como ya se ha mencionado desde el principio de este trabajo, resulta sumamente importante tomar en cuenta el aspecto afectivo ligado a lo cognitivo, porque la vanguardia tecnológica por sí sola y sin la guía de un docente calificado y dispuesto a fomentar un ambiente positivo de interacción, muy difícilmente logrará que los estudiantes se conviertan en aprendices autónomos: "affect is a means to an end, or, said differently, affect is the mediator between a number of teacher communications variables and cognitive learning" (Rodríguez et al., 1996, p. 303). Al respecto, el Marco Común Europeo de Referencia para las lenguas (en adelante MCER), recomienda 
tomar en cuenta factores como la reflexión, el análisis, el descubrimiento, y ante todo, la acción e interacción del estudiante. Esta perspectiva acentúa y posiciona el papel de la lengua como un instrumento mediante el cual se debe incentivar al alumnado a resolver situaciones comunes de su quehacer diario con los demás, es decir, a que más allá del uso de la comunicación se favorezca la acción (Herrera y Conejo 2009, p. 2).

Por esta razón, al hacer de nuestros alumnos aprendientes autónomos centrados en su propio aprendizaje, es de esperarse que el ambiente en clase se torne positivo y que la dinámica de trabajo fluya mejor. Sin embargo, no solamente es necesario desarrollar un ambiente afectivo óptimo dentro de la clase, sino fuera de ella también. Según Jane Arnold (2001, p. 71) "para facilitar el aprendizaje efectivo y afectivo en las clases de lengua hay que despertar sensaciones, reflexiones, pasar a la acción con estrategias cooperativas y tareas enriquecedoras", por lo que el docente de lenguas tiene un nuevo reto al que enfrentarse en el siglo XXI: hacer que el contenido tecnológico sea capaz de llamar la atención de sus estudiantes.

Retomando nuevamente a Caballero (2009, p. 2), el camino hacia el mundo de la digitalización en el ambiente educativo tiene sus raíces a partir de cambios socioculturales surgidos en el mundo a partir de las últimas décadas, tales como la globalización, la virtualización de los procesos sociales y el uso de las TIC. Asimismo, es importante señalar la declaración de principios de la Cumbre Mundial de la Sociedad de la Información (CMSI), pues es precisamente donde se comienza a construir el discurso de la nueva maquinaria tecnológica que nos ha llevado a buscar respuestas a problemas comunes y a compartir el conocimiento bajo una "conciencia planetaria"; o dicho de otra forma, el mundo se vuelve un lugar más interconectado en donde los problemas que antes afectaban a las naciones por separado, ahora nos atañen a todos por igual (Caballero, 2009, p. 3). Por lo tanto, uno de los principales aportes de las TIC es su capacidad de trascender los límites espacio-temporales, situación idónea para forjar ambientes de aprendizaje autónomos en el campo de la educación. Sin embargo, el primer paso a dar es la alfabetización digital que Casado (2006) define como "poseer la capacitación imprescindible para sobrevivir en la sociedad de la información y poder actuar críticamente sobre ella" (p. 68). Así pues, mientras más se logre establecer una cultura de alfabetización digital en los docentes, mejores serán los beneficios para el alumnado de las diversas instituciones educativas alrededor del mundo.

La incorporación de las TIC en la enseñanza de LE ha sido parada obligatoria para muchos de nosotros en las últimas tres décadas. Gracias a ello no solo hemos sido testigos de las constantes actualizaciones de software y hardware en nuestros centros educativos y ambientes personales, sino que también hemos tenido la oportunidad directa de ver cómo las habilidades, aptitudes, y forma de ser de nuestro alumnado ha ido cambiando con el paso del tiempo; sobre todo en el caso de los docentes con más años de experiencia. Adell y Castañeda (2012, p. 14) explican que el propósito principal de la revolución digital ha sido "que los centros educativos preparen a los alumnos para un nuevo tipo de sociedad, la sociedad de la información, no solo enseñándoles a usar las TIC, sino también usándolas como herramientas de aprendizaje”. Estos mismos investigadores están conscientes de las consecuencias suscitadas a raíz de los cambios socioculturales y sus repercusiones en el contexto educativo; de ahí que propongan el concepto de una "pedagogía emergente", que nace con las TIC y que está anclada en enseñanzas importantes de la pedagogía del siglo XX. En términos concretos, no se trata de desechar los recursos analógicos y metodologías con los que hemos llevado a cabo nuestra labor docente por tantos años, sino expandir nuestros horizontes y estar abiertos a un "conjunto de enfoques e ideas pedagógicas, todavía no bien sistematizadas, que surgen alrededor del uso de las TIC en educación y que intentan aprovechar todo su potencial comunicativo, informacional, colaborativo, interactivo, creativo e innovador en el marco de una nueva cultura del aprendizaje" (Adell y Castañeda, 2012, p. 15). Por ende, entre más dispuestos estemos los docentes a invertir tiempo en adquirir un adecuado nivel de competencia digital, mejores oportunidades de detonar un proceso de enseñanza-aprendizaje basado en un enfoque transdisciplinario.

El empleo de las TIC ofrece una plétora de ventajas y habilidades como "el acceso desde áreas remotas, la flexibilidad en tiempo y espacio para el desarrollo de las actividades de enseñanza-aprendizaje o la posibilidad de interactuar con la información por parte de los diferentes agentes que intervienen en dichas actividades" (Ferro et al., 2009, p. 4). En definitiva, las TIC han llegado para quedarse; no incorporarlas en el día a día de nuestra profesión solo evidenciará una reticencia cada vez más acentuada de nuestros alumnos a no poner atención al contenido de las clases por estar distraídos con sus redes sociales, a completar ejercicios de conjugaciones verbales a través del relleno de huecos, a memorizar listas interminables y descontextualizadas de vocabulario al final de las páginas del libro, y a participar en pruebas y exámenes orales de preguntas y respuestas. Todos estos inconvenientes pueden acrecentar más la ansiedad lingüística que "es uno de los mayores obstáculos para el aprendizaje de idiomas y supone un reto importante en la actualidad tanto para los profesores de lenguas extranjeras como para los investigadores en este caso" (Vieco, 2019, p. 11).

Por todo lo anterior, se hace patente la necesidad de ir más allá de los "hábitos lingüísticos" que por mucho tiempo han dominado el escenario de la metodología de LE, y que pensaríamos que gracias al enfoque comunicativo hoy serían tan solo un vestigio del pasado (Baralo y Estaire, 2010, p. 214). Es imposible negar que estamos ante un cambio radical en el campo educativo, por lo que obligar a nuestros estudiantes a 
aprender de la misma forma en que muchos de nosotros lo hicimos tiempo atrás, hoy por hoy, simplemente resulta inocuo. Continuar anclados en enfoques tradicionales y sin reflexionar del porqué de ello, solo ocasionará que los alumnos pierdan la motivación necesaria para continuar aprendiendo y que los docentes no sepamos aprovechar las oportunidades que las TIC nos ofrecen.

\section{HACIA LA RENOVACIÓN DE LAS TIC : TECNOLOGÍAS DE LA RELACIÓN, INFORMACIÓN Y COMUNICACIÓN (TRIC)}

Hasta el momento nos hemos enfocado en la evolución y en las razones por las cuales debemos incorporar las TIC en el entorno educativo, particularmente en el proceso de enseñanzaaprendizaje de una LE. Hemos visto que para lograr un ambiente colaborativo en clase es necesario que los docentes asumamos la responsabilidad de sumarnos al proceso de alfabetización digital por medio de las TIC, y de esta manera acoplarnos mejor a las necesidades del nuevo aprendiente digital del siglo XXI. También, se ha hecho necesario tornar nuestra mirada a la dimensión afectiva en cuanto a su capacidad de forjar espacios positivos de aprendizaje y libres de emociones negativas, como la ansiedad lingüística que puede interferir en el proceso de desarrollo y evaluación de la expresión oral.

A lo largo de las últimas décadas han sido cuantiosos los estudios e investigaciones referentes al factor afectivo en el proceso de la enseñanza y aprendizaje de lenguas extranjeras (Stern, 1983; Rodríguez, Plax y Kearney, 1996; Underhill, 2000; Williams y Burden, 1999; Schumann, 2000; Arnold, 2000; Fonseca 2002; Dörnyei y Murphey, 2003). Por todos ellos, se ha podido constatar que las emociones están relacionadas estrechamente con los procesos cognitivos y que el docente debe ser consciente de fomentar un clima positivo de aprendizaje para evitar que la ansiedad se apodere de los estudiantes, ya que "si queremos que aprendan de forma eficiente hay que hacer que se sientan lo más cómodos posible" (Vieco, 2019, p. 2). De hecho, la ansiedad como un factor que incide en el aprendizaje de lenguas extranjeras llevó a que Horwitz et al., (1986) desarrollaran una escala para medir la ansiedad en lo que se conoce como "Foreign Language Classroom Anxiety Scale (FLCAS). Por su parte, Krashen y su teoría del filtro afectivo demuestra que "when the filter is up, input can't reach those parts of the brain where acquisition occurs" (p. 176). O dicho en palabras de Vieco (2019), "los estudiantes cuyas emociones no son buenas para la adquisición de una nueva lengua construyen un filtro psicológico que, aunque les permite captar el sentido del mensaje, impide que la información llegue a la región del cerebro responsable de procesar esa información lingüística" (p. 6). Por lo tanto, es primordial tener en cuenta que un curso de LE debe pensarse y diseñarse con sumo cuidado para presentar al estudiante actividades relevantes que potencien su desenvolvimiento.
Dadas todas estas condiciones, y según los teóricos españoles Marta-Lazo y Gabelas (2016), hemos llegado a un momento donde los seres humanos estamos "conectados en un ecosistema comunicativo y relacional" (27), palabras que implican centrar nuestra atención en el aspecto de las relaciones interpersonales, y no solamente en cómo se obtiene y divulga la información. Por esta razón, Aranda et al., (2012) proponen el Factor R-elacional con un doble propósito. Por un lado, se trata de ir más allá del reduccionismo tecnológico TIC; y por otro, de abordar la conexión entre sí de todos los elementos que integran las siglas a través del Factor "R", y para ello proponen las Tecnologías de la Relación, Información y Comunicación (TRIC). Bajo esta perspectiva, se menciona que si bien el factor R-elacional se manifiesta en varias disciplinas, es en el campo de la educación y la pedagogía donde adquiere mayor relevancia y solo con él se potenciará un entorno verdaderamente colaborativo y cooperativo. Al respecto, y como manera de adaptarlo a la enseñanza y aprendizaje de ELE, se presenta en el siguiente apartado una actividad didáctica que toma en cuenta el factor R-elacional a través de tres espacios que lo componen, tal y como lo señalan MartaLazo y Gabelas (2016, p. 95): cognitivo, emocional y social.

\section{PROPUESTA DIDÁCTICA POR MEDIO DE LAS TRIC: REALIZACIÓN DE VIDEOS CORTOS EN LA CLASE DE ELE}

Con el arribo de los teléfonos inteligentes, vino también el uso de las cámaras de video que en los últimos años no solamente han revolucionado el entorno personal de los seres humanos, sino el educativo. La presente propuesta didáctica consiste en la realización de videos cortos en los cursos de ELE por parte de estudiantes matriculados en los niveles básico (SPA100Y), intermedio (SPA220Y) y avanzado (SPA320Y) del departamento de español y portugués de la Universidad de Toronto, Canadá. El nivel de correspondencia según el MCER para las lenguas comprende los niveles A1-B2. La propuesta tiene dos propósitos fundamentales. Primero, se trata de generar una dinámica de trabajo colaborativo donde los estudiantes tengan la oportunidad de expresarse oralmente en un ambiente menos rígido, como puede ser el caso de los tradicionales exámenes orales, y que muchas universidades suelen adoptar a partir de sesiones individuales de preguntas y respuestas del profesor al estudiante, o por medio de juegos de rol (role play) individuales o en equipos, y donde cada estudiante adopta un determinado papel o personalidad. El segundo propósito de esta actividad es evaluar, al final del año escolar, el nivel de competencia oral que los estudiantes hayan adquirido en el curso. Para ello se parte de un acercamiento que toma en cuenta la dimensión afectiva-emocional de la enseñanza y aprendizaje de LE como herramienta reductora del filtro afectivo, manifestado a través de la frustración y la ansiedad, ya que "de nada sirve enseñar gramática y léxico si no conseguimos que haya comunicación" (Vieco, 2019, p. 4). 
El programa de cursos de ELE en el departamento de español y portugués de la Universidad de Toronto cuenta con alrededor de 1000 estudiantes cada año, de los cuales la mayoría se concentran en el nivel básico (aproximadamente 600 estudiantes). A juzgar por los resultados preliminares de los videos, basados en algunos de los comentarios de estudiantes en las evaluaciones de fin de curso, así como las opiniones de los docentes a cargo de diferentes secciones de lengua, se revela que realizar videos cortos representa un valioso instrumento de enseñanza basado en el factor afectivo. De igual forma conviene hacer hincapié en algunos datos de tipo cuantitativo que representan de manera estadística la situación interna del departamento, y que a primera vista apuntan hacia una estabilización en el número de estudiantes que declaran una subespecialización en español, pese a observarse una ligera disminución. Este último dato es en sumo relevante y positivo si lo comparamos con la situación precaria actual por la cual atraviesan muchos de los programas y departamentos de lenguas extranjeras en el país y en los Estados Unidos, en donde se ha visto una marcada disminución de estudiantes. Si bien los datos estadísticos internos del departamento de español y portugués de la Universidad de Toronto no son concluyentes y/o quieren decir que se deba enteramente a la realización de videos cortos en la clase de ELE, sí se puede inferir que un estudiante que ha escogido declarar una subespecialización es porque, en mayor o menor medida, se ha sentido cómodo con los parámetros de evaluación, de entre los que destaca el componente oral y que suele ocupar de entre diez a veinte por ciento de la calificación total.

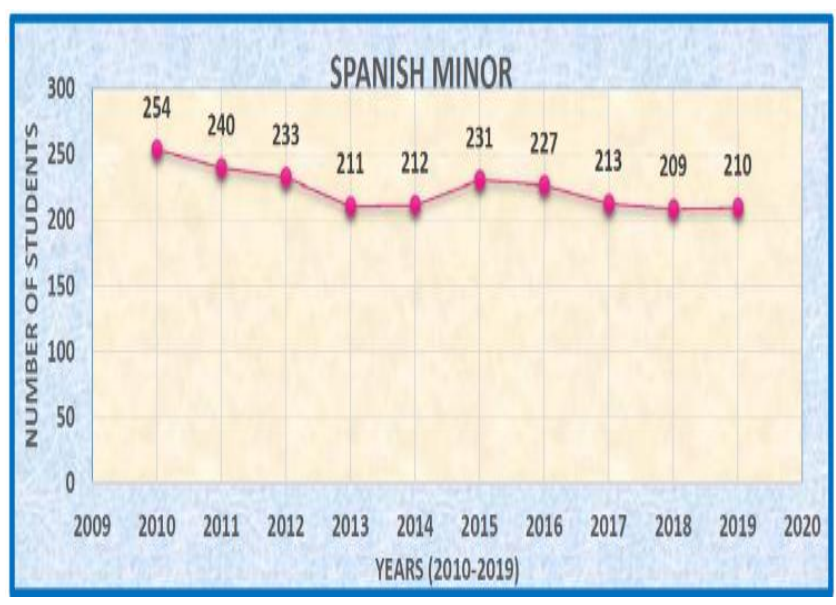

Gráfica 1. Estadísticas internas del departamento de español y portugués de la Universidad de Toronto 2010-2019.
Cabe mencionar que la realización de los videos cortos en las clases de ELE se organiza logísticamente en equipos de entre cuatro a siete personas, dependiendo del número de estudiantes matriculados en total. Como el curso para principiantes (SPA100Y) tiene una capacidad de 75 estudiantes, entonces los grupos no deben rebasar las siete personas. Para las clases de nivel intermedio (SPA220Y) y avanzado (SPA320Y), el límite de participantes es de cinco. Todos los cursos de lengua comienzan en el mes de septiembre y terminan en abril del siguiente año. La información sobre cómo llevar a cabo esta actividad es proveída en el programa de curso (syllabus) desde que comienzan las clases, documento donde también se adjuntan muestras de videos hechos en años anteriores y cuyo propósito es servir como fuente de inspiración. Además, el propósito de esta actividad también se comunica de manera explícita, incluidos los criterios de evaluación basados en cuatro categorías: creatividad, participación, claridad, vocabulario y gramática. Para asegurarnos de que todos hayan entendido los lineamientos y sepan exactamente lo que se espera de ellos para obtener una nota satisfactoria, la rúbrica de evaluación se provee en el idioma inglés.

Las categorías de evaluación presentadas en la rúbrica están pensadas desde el punto de vista del Factor R-elacional, como una manera de incorporar la dimensión afectiva al proceso comunicativo de la enseñanza-aprendizaje de una LE, y que no se vea limitado solamente por lo cognitivo (contenidos lingüísticos y funcionales), sino por lo emocional (creatividad), y lo social (participación de todos los estudiantes equitativamente). Y es en base a estos factores que tiene lugar el aprendizaje afectivo que, por doble partida, acerca a los estudiantes al aprendizaje de tipo experiencial; es decir, "a aprender haciendo" (Learning by doing), pues no solo se abre la puerta a la expresión oral, sino que en el trayecto se construye la capacidad de forjar vínculos emocionales entre todos los participantes y con ello potenciar los niveles de motivación y creatividad. Asimismo, estamos ante una oportunidad de "aprender a aprender" (Learn to learn), pues es posible que no todos los estudiantes tengan las mismas habilidades y/o competencia digital, como lo puede ser el manejo de la cámara, la creación de efectos especiales, y hasta la manera de incorporar subtítulos. Al trabajar de manera colaborativa y cooperativa, se fortalece el sentir de comunidad tan beneficioso en el proceso de aprendizaje de una LE, lo cual resulta imposible de replicar en un examen oral tradicional, tal y como señala Kremers (2000): "no sólo hay que enseñar cómo mantener el acto de hablar sino también cómo actuar en situaciones críticas. Y para ello es importante tratar la destreza como destreza comunicativa y productiva y ajustar los ejercicios más a ellos y a la situación fuera del aula" (p. 469). 


\begin{tabular}{|c|c|c|c|c|}
\hline \multicolumn{5}{|l|}{$\begin{array}{l}\text { Video Title: } \\
\text { Students: }\end{array}$} \\
\hline $\begin{array}{l}\text { Creativity } \\
\ldots / 25 \mathrm{pts}\end{array}$ & \begin{tabular}{|l|} 
Poor $(0-5$ pts $)$ \\
Students exhibited no \\
effort in the \\
assignment; \\
presentiation lacked \\
creativity
\end{tabular} & $\begin{array}{l}\text { Fair (6-11 pts) } \\
\text { Students barely met } \\
\text { the minimum } \\
\text { requirement of the } \\
\text { dssigninent; } \\
\text { presentation was not } \\
\text { completely creative }\end{array}$ & $\begin{array}{l}\text { Good (12-17 pts) } \\
\text { Students met the } \\
\text { requirement of the } \\
\text { assignment. } \\
\text { Assignniment was } \\
\text { mostly creative }\end{array}$ & $\begin{array}{l}\text { Excellent (18-25 pts) } \\
\text { Students exceeded } \\
\text { the basic } \\
\text { requirements of the } \\
\text { dassigntruent and the } \\
\text { project was highly } \\
\text { creative }\end{array}$ \\
\hline $\begin{array}{l}\text { Active Participation } \\
\text { _ } / 25 \text { pts }\end{array}$ & $\begin{array}{l}\text { Poor }(0-5 \text { pts }) \\
\text { Not all members } \\
\text { contributed to the } \\
\text { project, or one or } \\
\text { mure of the projetl } \\
\text { members contributed } \\
\text { inadequately to the } \\
\text { production }\end{array}$ & $\begin{array}{l}\text { Fair (6-11 pts) } \\
\text { All members } \\
\text { contributed modestly. } \\
\text { Some members are } \\
\text { fat less promintent in } \\
\text { the production than } \\
\text { others }\end{array}$ & $\begin{array}{l}\text { Good (12-17 pts) } \\
\text { All members } \\
\text { contributed to the } \\
\text { project, but some } \\
\text { members did nivt play } \\
\text { as significant a role in } \\
\text { the production as a } \\
\text { whole }\end{array}$ & $\begin{array}{l}\text { Excellent (18-25 pts) } \\
\text { All members } \\
\text { contributed equally in } \\
\text { the project }\end{array}$ \\
\hline $\begin{array}{l}\text { Clarity } \\
\ldots / 25 \mathrm{pts}\end{array}$ & $\begin{array}{l}\text { Poor }(0-5 \mathrm{pts}) \\
\text { Pronunciation is } \\
\text { largely } \\
\text { incomprehensible } \\
\text { ( } 50 \% \text { of words or } \\
\text { more). Intonation and } \\
\text { stress patterns often } \\
\text { nhstrust meaning }\end{array}$ & $\begin{array}{l}\text { Fair (6-11 pts) } \\
\text { Pronunciation of a } \\
\text { good number of high- } \\
\text { frequency words (75\% } \\
\text { or more) is } \\
\text { comprehensible to a } \\
\text { listener used to non- } \\
\text { native speakers. Main } \\
\text { intonation and stress } \\
\text { patterns (question, } \\
\text { statement, } \\
\text { exclamation) are } \\
\text { identifiable even with } \\
\text { a strong influence } \\
\text { frnm nther } \\
\text { languagels). }\end{array}$ & $\begin{array}{l}\text { Good (12-17 pts) } \\
\text { Pronunciation is } \\
\text { generally clear ( } 85 \% \\
\text { or more) even for } \\
\text { listeners not used to } \\
\text { non-native speakers. } \\
\text { Occasional } \\
\text { misprnnunciation } \\
\text { does not hinder } \\
\text { intelligibility. } \\
\text { Intonation and stress } \\
\text { patterns are used } \\
\text { adequately in simple } \\
\text { phrases, in spite of a } \\
\text { claar influenne from } \\
\text { other language(s). }\end{array}$ & $\begin{array}{l}\text { Excellent (18-25 pts) } \\
\text { Pronunciation is } \\
\text { almost totally clear } \\
\text { (95\% or more) for any } \\
\text { listener, even with a } \\
\text { noticeable influence } \\
\text { from other } \\
\text { language(s). } \\
\text { Occasional } \\
\text { mispronunciation of } \\
\text { less famillar sounds. } \\
\text { Intonation and stress } \\
\text { patterns are used to } \\
\text { support the meaning } \\
\text { of the message. }\end{array}$ \\
\hline $\begin{array}{l}\text { Grammar and } \\
\text { Vocabulary } \\
\\
\end{array}$ & $\begin{array}{l}\text { Poor }(0-5 \text { pts }) \\
\text { Words are less than } \\
10 \% \text { grammatically } \\
\text { correct. Lacking in } \\
\text { vocabulary }\end{array}$ & $\begin{array}{l}\text { Fair }(6-11 \text { pts }) \\
\text { Words are } 70-85 \% \\
\text { grammatically } \\
\text { correct. Showed some } \\
\text { vocabulary mastery }\end{array}$ & $\begin{array}{l}\text { Good (12-17 pts) } \\
\text { Words are } 85-95 \% \\
\text { grammatically } \\
\text { correct. Appropriate } \\
\text { vocabulary }\end{array}$ & $\begin{array}{l}\text { Excellent (18-25 pts) } \\
\text { Words are } 95 \% \text { or } \\
\text { more grammatically } \\
\text { correct. Excellent } \\
\text { display of varied } \\
\text { vocahulary }\end{array}$ \\
\hline
\end{tabular}

TOTAL: 100

Figura 3. Rúbrica de evaluación de videos

En cuanto al papel que juega el docente en esta actividad, podemos destacar su capacidad como facilitador y promotor de un aprendizaje autónomo. Por esta razón, la temática del video es a libre elección de los propios estudiantes, pues en todo momento se busca despertar la creatividad a través del factor R-elacional-emocional. Por ello se da la libertad a los estudiantes para que sean ellos quienes decidan qué escenario temático les parece mejor presentar. En este sentido, cabe recalcar también que el día de la evaluación todos los participantes deben estar presentes en clase para mostrar sus videos y así poder compartir con los demás sus creaciones. Bajo esta perspectiva, se busca también dotar a los partícipes de seguridad en sí mismos (autoconfianza), elemento indispensable en el aprendizaje de una LE.

Por último, es importante señalar que esta actividad no solamente sirve como telón de fondo para desarrollar la expresión oral y la competencia comunicativa, sino que también fomenta un espacio positivo de aprendizaje a través de los factores lúdico y competitivo. Para ello se ha creado la noche de los "Oscars" (Spanish 'Oscars'), un evento donde año con año se premia con dinero en efectivo el talento de los estudiantes. Para participar, solo es necesario mandar de manera electrónica el video creado en clase pero con subtítulos en inglés, ya que a la noche de los "Oscars" se invita al público en general y es posible que no todos tengan dominio del idioma español. La caracterización de este evento es de suma importancia y añade un toque especial; por eso se exhorta a los concursantes e invitados a portar sus mejores prendas de vestir. El jurado suele estar conformado por profesores del departamento y de otros departamentos de lengua de la universidad, así como de estudiantes graduados que cursan programas de maestría o doctorado y que al mismo tiempo fungen como asistentes de enseñanza. En definitiva, los Spanish "Oscars" es un concurso que ha sido bien recibido no solo por parte de la comunidad estudiantil, sino también por la comunidad hispanohablante local que se siente orgullosa y deseosa de poder fungir como fuente de contacto con la universidad y así fomentar la práctica del español en la ciudad de Toronto que, dicho sea de paso, se ha colocado como uno de los idiomas con mayor crecimiento en los últimos años.

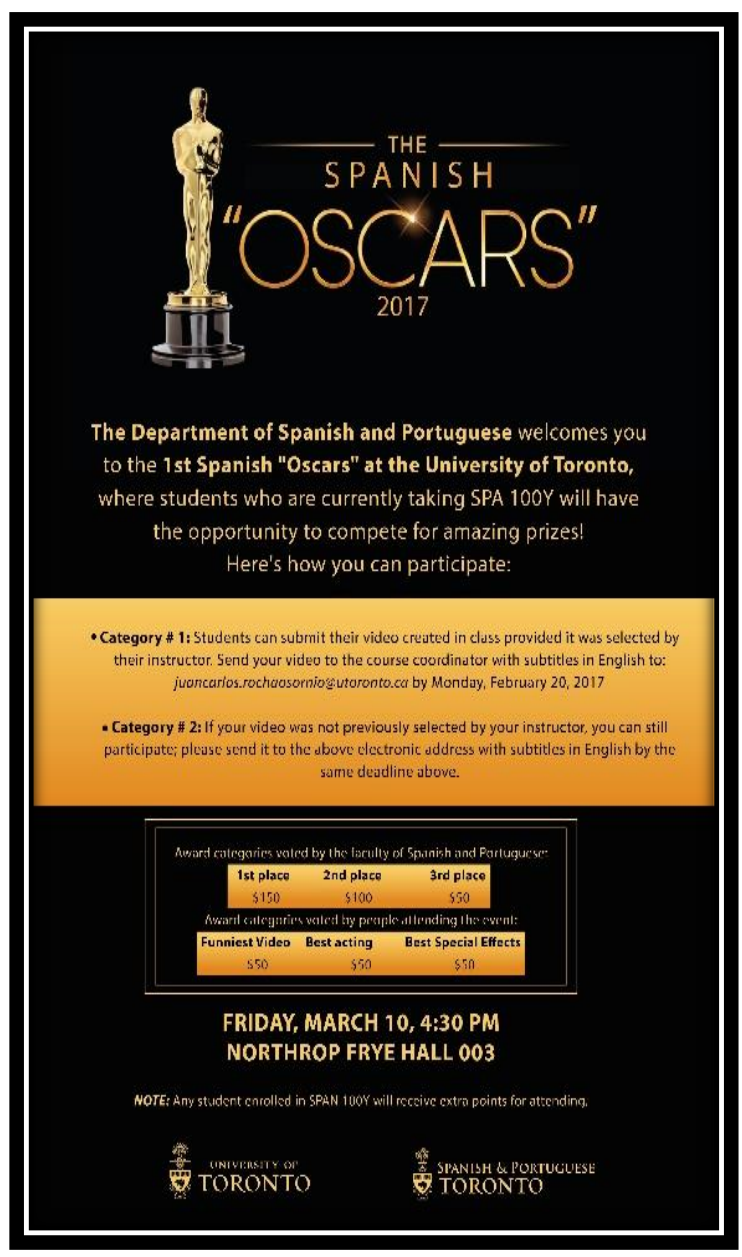

Figura 4. Afiche del evento Spanish "Oscars” 2017 


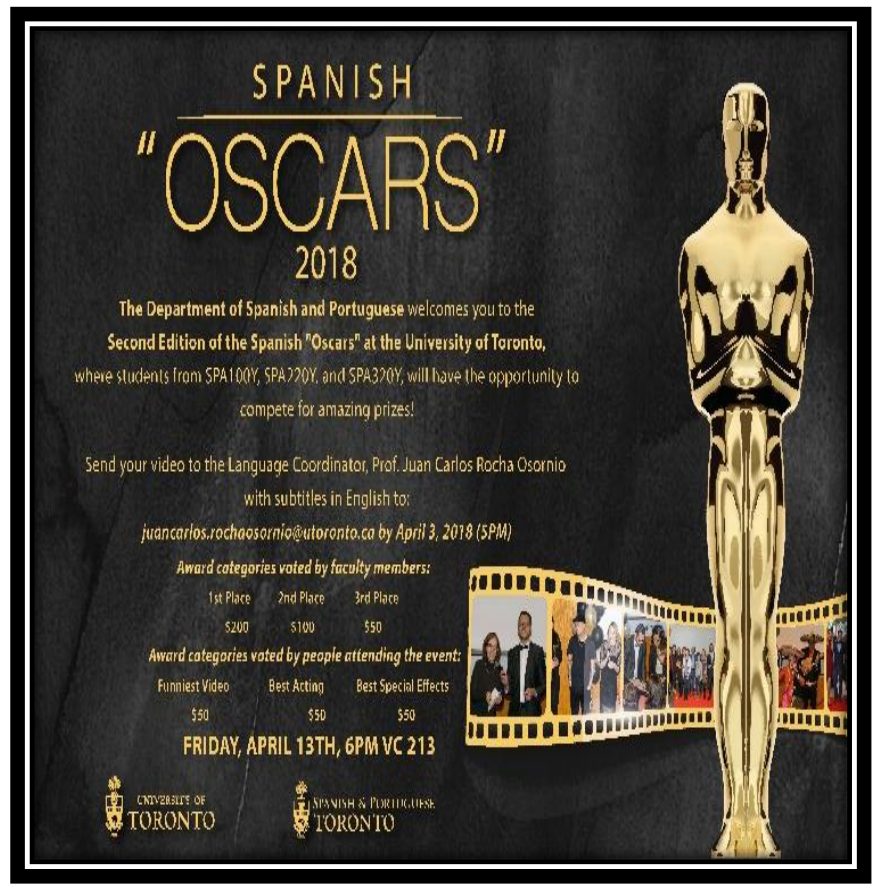

Figura 5. Afiche del evento Spanish “Oscars” 2018

\section{CONCLUSIONES}

En este artículo se ha puesto atención en presentar una manera alternativa e innovadora de desarrollar y evaluar la expresión oral en los cursos de ELE de la Universidad de Toronto. Para este fin, se ha optado por partir de los beneficios que ofrecen las Tecnologías de la Información y la Comunicación (TIC), los cuales se presentan renovados por un nuevo concepto: las Tecnologías de la Relación, Información y Comunicación (TRIC), hermanadas a través del Factor R-elacional y propuesto por Marta-Lazo y Gabelas (2012) desde tres aspectos: cognitivo, emocional y social. El resultado ha sido la creación de video cortos, y cuya implementación se apoya en la dimensión afectiva de la enseñanza-aprendizaje de una LE. La contribución de este trabajo, de carácter descriptivo y reflexivo, representa una manera de abordar y traer a la mesa de discusión el tema de la expresión oral, y buscar los mecanismos adecuados para tratar de reducir al máximo la ansiedad lingüística que puede causar estragos en el proceso de aprendizaje de una lengua extranjera. Dado que vivimos tiempos de grandes cambios en el plano educativo que requieren que los docentes nos embarquemos en un proceso de mayor compromiso con nuestro estudiantado, entonces es recomendable que seamos pacientes con nosotros mismos, que nos formémonos digitalmente, y que vivamos el tránsito de lo analógico a lo digital con emoción. Y sobre todo, que nunca perdamos de vista que una LE es una entidad viva llena de emociones, tal y como cuando le mostramos a un estudiante el cambio de significado entre una oración interrogativa y exclamativa, por ejemplo. Además, y por más avances tecnológicos que existan, los docentes seguimos al frente de nuestros grupos, pese a las injerencias del COVID19 que a muchos nos ha obligado a trabajar desde casa. Solo con el paso del tiempo veremos si la ciencia es capaz de reemplazarnos por la inteligencia artificial. Pero ese es otro tema del que seguramente estaremos todos muy pendientes y que habrá de aportar cambios importantes en el campo de la enseñanza de lenguas extranjeras.

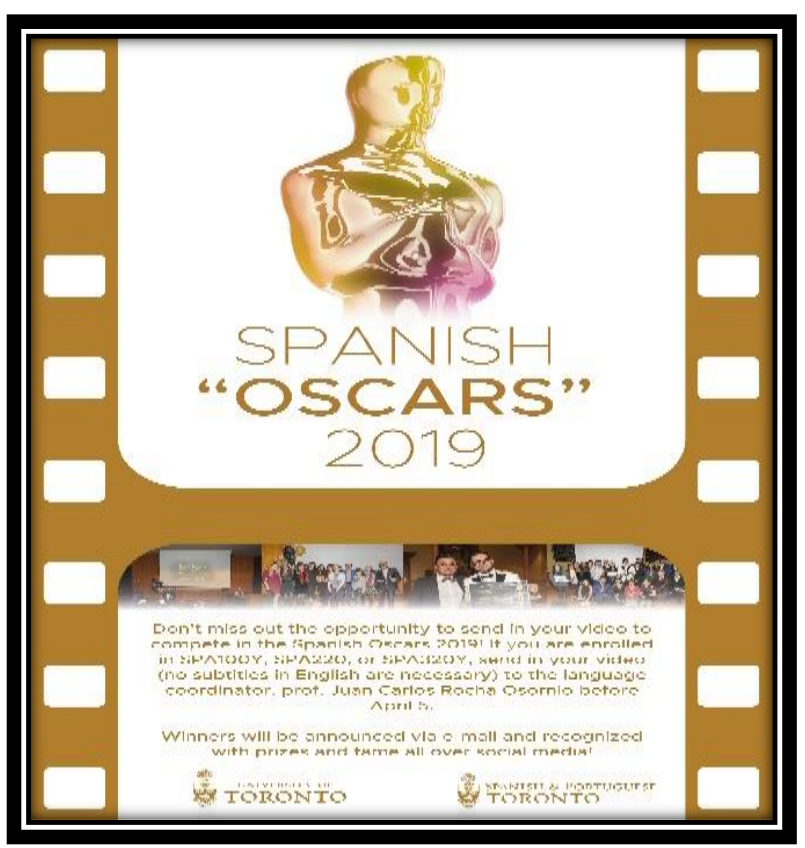

Figura 6. Afiche del evento Spanish "Oscars” 2019

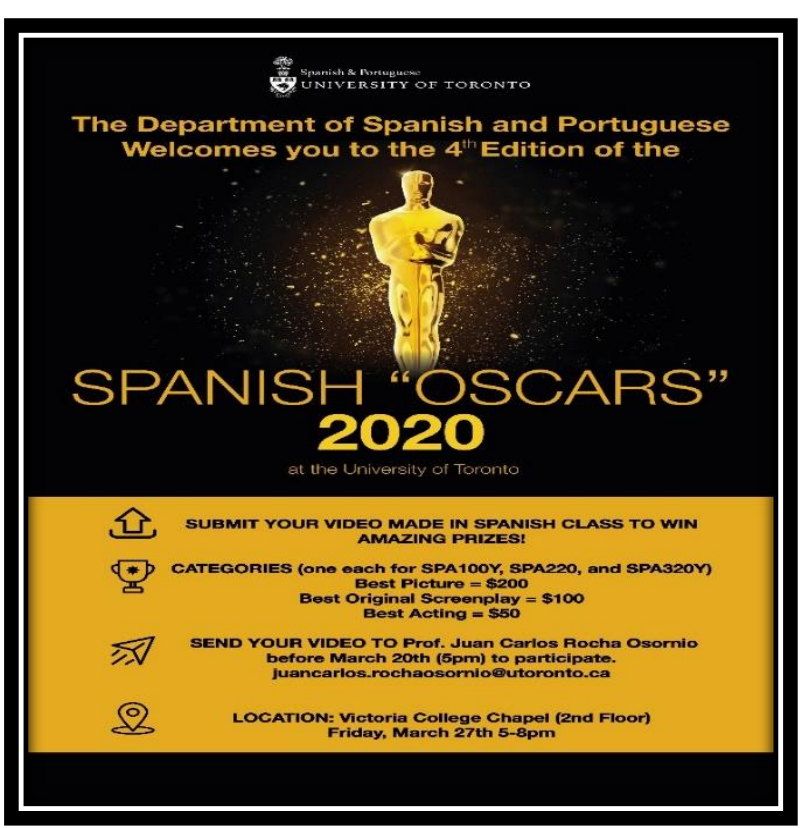

Figura 7. Afiche del evento Spanish "Oscars" 2020 que desafortunadamente se tuvo que cancelar debido a la irrupción de la pandemia por COVID-19. 


\section{REFERENCIAS}

[1] Adell, J., y Castañeda, L. (2012). Tecnologías emergentes, ¿pedagogías emergentes? En J. Hernández, M. Penessi Fruscio, D. Sobrino y A. Vázquez (Eds), Tendencias emergentes en educación con TIC (pp. 1332). Barcelona, España: Espiral.

[2] Aguilar, M., y Leiva, J. (2012). La participación de las familias en las escuelas TIC: Análisis y reflexiones educativas. Pixel-Bit. Revista de Medios y Educación 40, pp. 7-19. Recuperado de:

https://www.redalyc.org/articulo.oa?id=36823229001

Consultado el 16/06/2020

[3] Aranda, D.; Marta-Lazo, C. y Gabelas, J.A. (2012). Por qué las TRIC y no las TIC. COMeIN 9. Recuperado de:

https://www.uoc.edu/divulgacio/comein/es/numero09/articles/ArticleDani-Aranda.html

Consultado el 16/06/2020

[4] Arnold, J. (2000). La dimensión afectiva en el aprendizaje de idiomas. Madrid: Cambridge University Press.

[5] Arnold, J. (2001). Exploring aspects of group dynamics in holistic language learning. En Bruton, A. y Ramírez, B. (eds.) Lingüística Aplicada al Aprendizaje del Inglés. Sevilla: Grupo de Investigación Humanidades 125 .

[6] Arnold, J. y Fonseca, M.C. (2005). Multiple Intelligence Theory and Foreign Language Learning: A Brain-Based Perspective. IJES. International Journal of English Studies, 4(1), 119-136.

[7] Baralo, M., \& Estaire, S. (2010). Tendencias metodológicas postcomunicativas. En C. Abelló, C. Ehlers y L. Quintana (Eds) Escenarios biligües: el contacto de Lenguas en el Individuo y la Sociedad (pp. 105-128). Bern: Peter Lang.

[8] Caballero, S. (2009). Tránsito digital en el ámbito educativo. Revista Iberoamericana de Educación, 48(6), pp. 1-13.

[9] Casado, R. (2006) Claves de la alfabetización digital. Madrid: Fundación Telefónica y Foro de Investigación y Acción Participativa para el desarrollo de la Sociedad del Conocimiento (FIAP).

[10] Caro, C., y Plaza, J. (2016). Intervención educativa familiar y terapia sistémica en la adicción adolescente a internet: fundamentación teórica. Revista Española de Orientación y Psicopedagogía, 1(24), 99-113.

https://doi.org/10.5944/reop.vol.27.num.1.2016.17031

[11] Dörnyei, Z. y Murphey, T. (2003). Group Dynamics in the Language Classroom. Cambridge: Cambridge University Press.

[12] Echeverría, J. (2003). La revolución tecnocientífica. Madrid, Fondo de Cultura Económica.
[13] Espinar, R., y López, C. (2009). Jóvenes y adolescentes ante las nuevas tecnologías: Percepción de riesgos. Athenea digital 16. 1-20.

https://doi.org/10.5565/rev/athenead/v0n16.509

Consultado el 16/06/2020

[14] Ezeiza, A. y Palacios, S. Evaluación de la competencia comunicativa y social en foros virtuales. RELIEVE: Revista Electrónica de Investigación y Evaluación Educativa 15(2), 1-15. Recuperado de:

https://www.redalyc.org/pdf/916/91612906003.pdf

Consultado el 16/06/2020

[15] Fandos Igado, M. y Silvestre Puig, R. (2011). Servicios de microblogs en la enseñanza secundaria. Edutec. Revista Electrónica de Tecnología Educativa 38(a183), pp. 1-20

https://doi.org/10.21556/edutec.2011.38.384 Consultado el 16/06/2020

[16] Ferro, C., Martínez, A.I., Otero, M. C. (2009). Ventajas del uso de las TICs en el proceso de enseñanza-aprendizaje desde la óptica de los docentes universitarios españoles. EDUTEC: revista electrónica de tecnología educativa, 29, pp. 1-12.

[17] Hernández Mercedes, MdP. (2012): “La integración de las TIC en la clase de ELE. Panorama de una (r) evolución", Revista Internacional de Lenguas Extranjeras. Recuperado de:

https://revistes.urv.cat/index.php/rile/article/view/8

Consultado el 06/05/2020

[18] Herrera, F. y Conejo, E. (2009). Tareas 2.0: La dimensión digital en el aula de español lengua extranjera. Marco ELE Revista de didáctica español lengua extranjera. Recuperado de:

https://marcoele.com/descargas/9/herrrera_conejo.tareas2.0.pdf

Consultado el 07/05/2020

[19] Horwitz, E. K., Horwitz, M.B., y Cope, J. A. (1986). Foreign Language classroom anxiety. The Modern Language Journal. 70(2), 125-132. Iglesias, I. (2016). El perfil del profesor de español como lengua extranjera: dimensión formativa, dimensión técnica y dimensión afectivo-emocional. Studia Romanica Posnaniensia 43(3), 67-83.

[20] Instituto Cervantes (2006). Plan curricular del Instituto Cervantes.

https://cvc.cervantes.es/ensenanza/biblioteca ele/plan_curricular/

[21] Krashen, S. (1981). Second Language Acquisition and Second Language Learning. Oxford, Pergamon Press.

[22] Kremers, M.F. (2000). El uso de las estrategias de aprendizaje en la expresión oral. XI Actas del Congreso de la Asociación para la Enseñanza del Español como Lengua Extranjera. Recuperado de: https://cvc.cervantes.es/ensenanza/biblioteca ele/asele/pdf/11/11 0461 .pdf

Consultado el $17 / 06 / 2020$ 
[23] Levy, M., \& Stockwell, G. (2006). CALL Dimensions: Options and Issues in Computer-Assisted Language Learning. Mahwah, NJ: Lawrence Erlbaum Associates.

[24] Liu, L. (2020). La enseñanza de E/LE en línea ante emergencia de salud pública. Estudio de caso: China”, Marco ELE Revista de didáctica español lengua extranjera. Recuperado de:

$\underline{\text { https://marcoele.com/descargas/30/liu-ele en linea en china.pdf }}$

Consultado el 07/05/2020

[25] Marquès, P. (2000): Los medios didácticos. Recuperado de:

http://peremarques.pangea.org/medios.htm

Consultado el 04/06/2020

[26] MacIntyre, P., \& Gardner, R. (2006). Methods and Results in the Study of Anxiety and Language Learning: A Review of the Literature. Language Learning, 41(1), 85-117.

[27] Marta-Lazo, C. y Gabelas, J. A. (2016). Comunicación digital. Un modelo basado en el factor R-elacional. Barcelona: Editorial UOC.

[28] O’Sullivan, B; Weir, C; \& Horai, T. (2006). Exploring difficulty in speaking tasks: an intra-task perspective. The British Council: IELTS Research Reports 7(6), 119-160. https://www.ielts.org//media/research-reports/ielts rr_volume06 report5.ashx

[29] Pérez, A. (2010). Nuevas tecnologías: nuevas addicciones. Escuela de padres. Madrid: Gesfomedia.

[30] Prensky, M. (2001). Nativos digitales, inmigrantes digitales. On the Horizon 9(6). Recuperado de:

https://aprenderapensar.net/2009/05/18/nativos-digitales-vsinmigrantes-digitales/

Consultado el 16/06/2020

[31] Prensky, M. (2010). Nativos e inmigrantes digitales. Cuadernos SEK 2.0. Recuperado de:

https://www.marcprensky.com/writing/PrenskyNATIVOS\%20E\%20INMIGRANTES\%20DIGITALES\%20(SEK).pdf

Consultado el 16/06/2020

[32] Rodríguez, J. I., Plax, T. G., y Kearney, P. (1996). Clarifying the Relationship Between Teacher Nonverbal Immediacy and Student Cognitive Learning: Affective Learning as the Central Causal Mediator. Communication Education, 45(4), 293-305.

https://doi.org/10.1080/03634529609379059

[33] Román-Mendoza, E. (2018). Aprender a aprender en la era digital: Tecnopedagogía crítica para la enseñanza de LE/L2. Washington D.C: Routledge.
[34] Roncel Vega, VM. (2008). Autoconcepto, motivación y ansiedad en el aula de idiomas. Marco ELE Revista de didáctica español lengua extranjera. Recuperado de:

https://marcoele.com/descargas/7/roncel autoconcepto-motivacion.pdf

Consultado el 06/05/2020

[35] Schummann, J. (2000). Perspectiva neurobiológica sobre la afectividad y la metodología en el aprendizaje de segundas lenguas. En Arnold, J. (ed.) La dimensión afectiva en el aprendizaje de idiomas. Madrid: Cambridge University Press.

[36] Sloep, P., \& Berlanga, A. (2011). Redes de aprendizaje, aprendizaje en red. Revista Comunicar 37(19), 55-63.

[37] Stern, H. (1983). Fundamental Concepts of Language Teaching. Oxford: Oxford University Press.

[38] Tesouro, M. \& Puiggalí, J. (2004). Beneficios de la utilización del ordenador en el aprendizaje: un diseño experimental. Edutec-e: Revista Electrónica de Tecnología 17. 1-19.

[39] Underhill, A. (2000). La facilitación en la enseñanza de idiomas. En Arnold, J. (ed). La dimensión afectiva en el aprendizaje de idiomas. Madrid: Cambridge University Press.

[40] Veuthey, M. (2008). El estudiante ELE/L2 como aprendiente autónomo: desarrollo de la gestión del aprendizaje y del conocimiento. Jornadas de Formación del Profesorado en la Enseñanza de ELE y La Literatura Española Contemporánea. Recuperado de:

https://redined.mecd.gob.es/xmlui/bitstream/handle/11162/90939/0082 0113013674.pdf?sequence $=1$

Consultado el 16/06/2020

[41] Vieco, M. (2019). Las emociones contraatacan en el aula de español”, Marco ELE Revista de didáctica español lengua extranjera. Recuperado de https://marcoele.com/descargas/29/vieco-emociones.pdf Consultado el $11 / 05 / 2020$

[42] Vilchez, L. F. (2005). Estudio sobre la escuela. Promovido por FERE y Fundación SM. Madrid: SM.

[43] Williams, M. y Burden, R. (1999). Psicología para profesores de idiomas. Madrid: Cambridge University Press. 